\title{
MULTICRITERIA DECISION MAKING FOR PROJECT SCHEDULING UNDER RESOURCE CONSTRAINTS
}

\author{
Elena Rokou* \\ School of Mechanical Engineering \\ National Technical University \\ Athens, GR \\ E-mail: erokou@mail.ntua.gr \\ Konstantinos Kirytopoulos \\ School of Mechanical Engineering \\ National Technical University \\ Athens, GR \\ E-mail: kkir@mail.ntua.gr
}

\begin{abstract}
A holistic approach is proposed for defining the resource constrained project scheduling problem (RCPSP). The paper's aim is to give a formulation of the project scheduling problem where all deterministic aspects that have been previously explored in the relevant literature are covered. Our goal is to provide a way to model and solve project scheduling problems as they actually are, without compromises other than the assumption that the given inputs are realistic. In order to define the desired optimisation objectives, namely time, resource profile, robustness and cost and either generate a weight vector reflecting the decision makers preferences on the objectives or handling all of them as a single vector. the Analytic Network Process (ANP) (Saaty, 1996) was used. The result of this process is the definition of relative preferences among the optimisation objectives, in the form of a normalised weight vector that will be given as input to the next stage of the solution process, that is a multi-objective genetic algorithm. This a priori definition of the preferences has been proven more efficient than the popular process of solving the problem, computing the objective functions and then limiting the solution space based on the decision maker's preferences.
\end{abstract}

Keywords: ANP, project scheduling, MCDA

\section{Introduction}

Scheduling problems are been investigated since the late fifties, motivated by the need to improve and facilitate project management. Project scheduling is a complex problem that every project manager faces in the beginning of each project and the consequences of an ill designed schedule can seriously endanger the successful project execution and completion. Applications can be found in diverse industries such as construction management, software development, etc. In addition, project scheduling is very attractive for researchers, mainly those related to operational research, because the models in this area are rich and, hence, difficult to solve.

Project scheduling involves the development of a project base plan (baseline schedule) which specifies for each activity the precedence and resource feasible start and completion dates, the amounts of the various resource types that will be needed during each time period and as a result the corresponding budget required for the execution of the project (Brucker et al., 1999). The fundamental issue for relevant problems is to generate a schedule that is precedence and resource feasible that is to fulfill the initially set precedence constraints and respects the available capacity of the resources involved. But, apart from this major issue, it is also desirable to come up with a schedule of minimal total project duration and cost, smooth profiles for the resource types used and increase robustness, in order to minimize the effect of

\footnotetext{
${ }^{*}$ Corresponding author
} 
possible perturbations in the duration of the activities and the resource availabilities during the execution. Project managers depending on the project and the situation at hand give more or less importance to each of the above objectives, therefore, a multidimensional approach is implicitly or explicitly used in practice (Viana and Pinho de Sousa, 2000). These different aspects, are often conflicting and all of them need to be taken into consideration as they play different roles in the schedule generation process based on the specific organization and its priorities, the size and the budget of the project, the customer and other environmental parameters.

Despite that the project-scheduling problem, was initially faced as a "hard" problem assuming that it is fully observable, governed by well-defined laws of behavior and closed to the environment, this is not the case. Still, when trying to take into consideration all the parameters defining and affecting a good project schedule a very complex system emerges. Therefore, it is essential to try to define the project scheduling problem taking into consideration conflicts and uncertainties but in a level of abstraction that will keep it general and permit its modeling and solution.

The rest of this paper is organised as follows. In section 2 a brief overview of project scheduling subject to resource constraints, is presented. In section 3 the proposed model is in depth analysed. Finally, in section 4 conclusions of the conducted study and possible directions for further research on this subject are discussed.

\section{Literature Review}

In the resource constrained project scheduling problem we have a single project consisting of $\mathrm{n}$ activities plus a dummy source activity representing the "project start" and a dummy sink activity representing the "project end", both with zero duration and resource demands. There are two kinds of constraints, precedence and resource related. First, the activities should be processed in a specific order given by the precedence constraints, where each activity $i$ should start after the completion of all its immediate predecessors. Second, performing an activity requires resources, which have limited capacity. We have a set of $K$ resource types, and each resource type has a limited capacity $R_{k}$. Each activity $i$, in order to be processed, requires $r_{i k}$ units of resource type $k$. The activities are assumed not preemptive, thus their processing cannot be stopped once it has been started. All data is assumed to be deterministic and known in advance.

While the RCPSP as given above is already a powerful model, it cannot cover all situations that occur in practice. Therefore, many researchers have developed more general project scheduling problems, often using the standard RCPSP as a starting point. Generalizations of the activity concept, precedence constraints and network characteristics as well as extensions of the resource concept and multiple objectives have been proposed the last few years (Hartmann and Briskorn, 2010). The most commonly used variations and extensions are summarized in Table 1.

\begin{tabular}{|l|l|}
\hline RCPSP Extensions/Variation & References \\
\hline Activities can be split during their execution & $\begin{array}{l}\text { (Bianco et al.; Boctor, 1996; Damay et al., 2007; } \\
\text { Demeulemeester and Herroelen, 1996; Peteghem and } \\
\text { Vanhoucke, 2010; Talbot, 1982) }\end{array}$ \\
\hline Multiple modes of execution & (Alcaraz et al., 2003; Boctor, 1996; Brucker et al., \\
& $\begin{array}{l}\text { 1999; Buddhakulsomsiri and Kim, 2006, 2007; Coelho } \\
\text { and Vanhoucke, 2011; De Reyck and Herroelen, 1999; }\end{array}$ \\
& $\begin{array}{l}\text { Deblaere et al., 2010; Heilmann, 2001, 2003; Jarboui et } \\
\text { al., 2008; Józefowska et al., 2001; Kolisch and Drexl, }\end{array}$ \\
& $\begin{array}{l}\text { 1997; Kyriakidis et al., 2012; Lorenzoni et al., 2006; } \\
\text { Mika et al., 2005; Mika et al., 2008; Peteghem and }\end{array}$ \\
& Vanhoucke, 2010; Sabzehparvar and Seyed-Hosseini, \\
& 2008; Seifi and Tavakkoli-Moghaddam, 2008; Ulusoy \\
& et al., 2001; Voß and Witt, 2007) \\
\hline Variable resource request and availability & (Hartmann, 2013) \\
\hline
\end{tabular}


In addition to the parameters previously analysed there are also various alternative optimisation objectives, although the minimisation of the duration of the project is the most popular one. These objectives can be classified as: time based, robustness based, objectives for rescheduling and objectives based on renewable resources and non-renewable resources consumption and smoothness of profiles.

Besides the objective of minimising the duration one may consider other objective functions depending on the completion times of the activities, like the total flow time or more generally the weighted (total) flow time. Other objective functions depend on due dates, which are associated with the activities, as the maximum lateness and the total tardiness, Ballestin et al. (2008), Kolisch (2000), and Viana and de Sousa (2000) consider the minimisation of the weighted version of this objective. All the above objective functions are regular, thus monotone non-decreasing in the completion times. On the other hand, objectives like the maximum earliness are an example for a non regular objective function (Lorenzoni et al., 2006).

Another non-regular objective function that is quite commonly used (Kimms, 2001; Mika et al., 2005; Vanhoucke et al., 2008) deals with the net present value, where a so-called cash-flow is associated with each activity and it is supposed to occur at the completion time of that activity. The objective is to maximise the net present value (NPV) given a discount rate $\alpha \geq 0$.

Resource based objectives occur in the area of resource investment (RIP) and resource levelling problems (RLP). In the RIP (Neumann et al., 2003)the resource capacities $R k$ are not given but have to be determined as additional decision variables, given the per unit cost of each resource type $c k$ and a target value of resources to be used $Y k$, the objective is to find a schedule with makespan less than the given project deadline $T$ and minimal resource cost.

In the RLP, the variation or the deviation of the resource usage over time is measured. In the deviation problems given a resource profile, the goal can be (Davis, 1973; Neumann and Zimmermann, 2000)to minimise the deviation from a given resource usage level, the overload or the squared deviation.

On the other hand, in variation problems the resource usage should not substantially vary over time. This can be achieved by minimising the per period variation, the max variation, or the squared per period variation. During the execution of a project, delays may occur that could not have be foreseen when the schedule was determined. Therefore, a project manager might be interested in a robust schedule that is a schedule in which a delay has only a limited effect. This approach is often referred to as proactive scheduling (Abbasi et al., 2006; Kobylański and Kuchta, 2007).

Rescheduling is necessary if the project is already in progress, but due to unexpected events (e.g., delays) the schedule that has been calculated before the start of the project is no longer valid. In such a situation, the problems characteristics may have changed. For example, some activities may already be finished and can be ignored, other activities may be in progress and must be considered unchangeable and the resource availability may have changed and might even have switched from time-independent to time-dependent. In contrast to proactive scheduling which anticipates disruptions by building robust schedules, here the case is that some disruption has already occurred and a new schedule has to be determined with minimal differentiation from the original/baseline schedule. This case is often referred to as reactive scheduling (Calhoun et al., 2002; Vanhoucke et al., 2001).

The standard RCPSP problem has numerous variations and extensions but not a total model that will 
include all those parameters that really matter when scheduling a real-world problem. This research gap along with the need for multiple objectives when scheduling a project induced the proposed research.

\section{Proposed Approach}

The proposed approach consists in defining the desired optimisation objectives, namely time, resource profile, robustness and cost and generating a weight vector reflecting the decision makers' preferences on the objectives. To weight the objectives, the Analytic Network Process (ANP) (Saaty, 1996), which is a generalisation of the Analytic Hierarchy Process (AHP), was used.

The reasons behind the selection of the specific method instead of other MCDA methods reside on its basic characteristics: simplicity, handling of mixed qualitative and quantitative inputs versus exclusively quantitative methods, no need of an analyst to support the process and focus on the subjective perspective of the decision maker to the problem.

In this section the ANP method is described and a generic model for weighting project-scheduling objectives is provided. The result of this process is the definition of relative preferences among the optimisation objectives, in the form of a normalised weight vector that will be given as input to the next stage of the solution process. This a priori definition of the preferences has been proven more efficient than the popular process of solving the problem, computing the objective functions and then limiting the solution space based on the decision maker's preferences.

In this multi-criteria decision analysis method, the first step is the identification of the criteria and the alternative solutions of the problem to be solved. Then, a graph structure, the so-called network, is created and the decision maker is asked to pairwise compare the components, in order to determine their priorities.

The decision about whether AHP or ANP should be used is based on the problem being solved and the corresponding network structure. The network is a logical conceptualization of the problem that reduces it, to its essentials. When the elements and their connections are easily located in levels of dominance with connections that transmit influence downwards, a hierarchical structure is best fitted for the decision problem. On the other hand, if the elements and their connections are complicated and can only be grouped in clusters that do not fit well in defined levels, a network structure is more appropriate.

The first step consists in the identification of the decision alternatives, goals and elements, where as elements are defined the criteria used to rank the different alternatives based on the preferences of one or more decision makers that constitute the decision making group. These criteria derive from the purpose of each functional unit, of the system in question. The project-scheduling problem has as alternative solutions the optimisation objectives that have been chosen in the previous stage, therefore the elements:

- Duration that refers to the makespan of the schedule and respect of deadlines set for certain activities or phases of the project

- Cost as the sum of resource and non resource related costs generated by a specific schedule,

- Resource Profile, which refers to the smoothness of one or more renewable resource types profiles as they are utilised in the schedule,

- Robustness that is about the total free slack in the generated schedule and it is used to enhance the stability of the schedule in case of alterations: in the duration of one or more tasks, the resource availabilities and/or requirements.

- Max Resource Usage, which is used to limit the maximum usage of expensive or rare resource types. 
Beside the alternative solutions of the problem, in the network are included all the criteria that will be used to rank the objectives which are also defined as elements. The identification of the criteria is an iterative process where all the decision makers related to this problem need to discuss which are the factors that affect the definition of an objective as more important than another taking into consideration the organisational strategy, the current situation and characteristics related to the project itself and the customer related to it. Therefore the criteria are subject to change from project to project and time to time. However, here a generic set of criteria that can be widely used, is provided. The selection of the criteria is based on interviews with experts of the project management field. The proposed set of criteria can be summarised in the following elements:

- Budget: the budget available for the project is a good indicator of how important will be the cost factor in the decisions to be taken, as a tight budget will let small margins for those makespan minimisations that require high level of resource availability and usage, or expensive resource types. On the other hand it complies with the minimisation of the maximum resource usage and the smoothness of resource profiles however, it is not strogly related to the objective of robustness.

- Cash Flows: timing of the inflows can affect the overall strategic decisions about the project, for example when the inflows are attached to specific milestones, then the deadlines, therefore the time objective, is critical for the whole project's viability. Similarly, the outflows affect the ranking of the objectives, as for example large payments connected to specific activities can lead to right shifting those activities and thus slowing down their execution.

- Resource Types- Cost, Contact type: the cost of the resource types to be used and the type of contractual relation that they have with the company that is executing the project affect a variety of decisions about the way that the project should be executed and how the objectives should be ranked. Permanent contractual relations, ask for very smooth resource profiles and enforce the need to be within the given resource requirements as the need for new resources will call for hiring of probably, temporary personnel, with unsure impact on the efficiency of the work team. Low daily rates and extended usage of external contractors lead to flexibility of the resource related constraints and low ranking of the resource profile objective. The existence of different resource types and associated needs for their profile is handled using different weights for each resource type within the resource profile objective.

- Penalties/Bonuses: in case of penalties and/or bonuses related to the completion time of the project or specific milestones, the time objective is expected to be prioritised and the cost objective needs to be balanced in relation to the time related loss or gain of money.

- Project's Contract: is related to specific clauses of the contract signed between the organization and the customer which might affect the ranking of the objectives. For example in case that there are clauses specifying quality levels of the generated project outcome/product then the robustness objective's value is elevated due to the need to have extra time available to anticipate the need to rework part of some activities to meet the required quality levels.

- Legislation: related to work hours, hiring and firing of personell, subcontracting, defining wages, specialised personnel needed to execute specific activities, environmentall issues, etc. affects the way that the project should be scheduled and thus the ranking of the objectives.

- Synergies: existing projects, on going or already executed, can be combined to the under examination project in terms of budget, human resource pool, equipment, etc., affecting this way the priorities set for the specific project, especially, the aspects of cost and resource usage.

- Resource Availabilities: refers to the effort needed to find extra resources if needed, the corresponding cost of using extra units of some resource type and when referring to work force, the expected efficiency of the new resources when combined to the existing ones. This data are always related to circumstantial factors as the specific time period, the social and political situation, etc. Having low resource availabilities makes the resource constraints less flexible and 
the possibility to use execution modes of activities requiring large numbers of resources very expensive, thus unlikely.

- Risk: refer to scope risks, like ill defined scope, integration issues, scope creep, etc., schedule risks as delayed decisions, wrong estimations of duration and effort needs, ommision of dependencies, etc. and resource related risks as delays caused by outsourcing, lack of cash flow, low quality and/or attrition of resources, loss of work team balance by people joining the team late, scarcity of skills. The types and levels of risk are strongly related to all the objectives and based on the probability of appearance and the expected impact of each one, different objectives can be prioritised for the mitigation of one or more risks.

- Expected Benefits: are related to factors like the project's expected outcomes and the customer's importance for the organization. The expected benefits can balance high costs and prioritize objectives like time and robustness.

The second step consists in the categorisation of the elements in suitable clusters that is based on the similarity of the previously identified element's characteristics. The criteria and alternative solutions composing the model for ranking the project scheduling objectives, are grouped in three clusters: Organisational containing the criteria that are strongly related to the specific organisation, its strategy and profile, Financial, containing the cost and budget related factors and Other, containing the factors that could not fit in the previous two categories. Finally, there is also the Alternatives cluster containing the objectives to be ranked.

Then, the definition of the influences takes place: inner dependecies reffer to influences (social, political, technical, economic, etc.) between two elements of the same cluster and outter dependencies between two different clusters. In this model, the Alternatives cluster depends on all the other clusters and viceversa. The Financial cluster influences and is influenced by the Organisational, as the resource availability influences the rates, the budget is affected by the risk factors, the synergies and the expected benefits, and there are risks related to the flows and the budget. The Organisational cluster is influenced by the Others cluster and it has inner dependecies among its components, as the legislative rules and the contract affect the risk factors, for example having high penalties for exceeding the deadline or getting products below the expected quality level raise the impact of the corresponding risks. The penalties along with the specific project contract influence the expected benefits. As concerned to the inner influence for the Organisational cluster, the synergies and the risks influence the expected benefits, the more synergies that can be generated through the project and the less risk the higher are the expected benefits. The final model is shown in Figure 1.

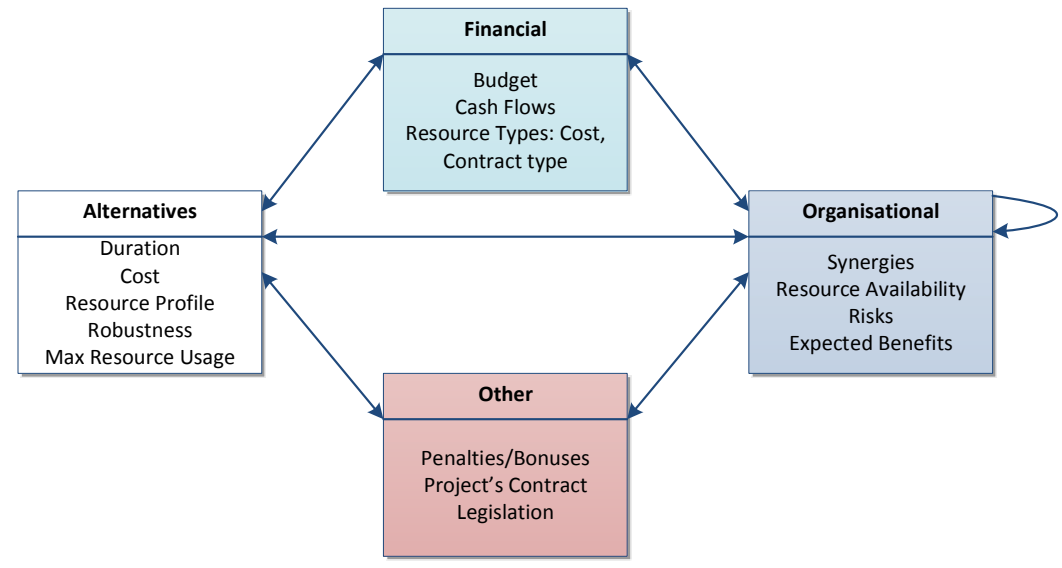

Figure 1 ANP model for project scheduling 
The fourth step consists in the forward and backward examination of the network by cluster, in order to make sure that the generated network structure is complete and consistent. The decision makers check that the network reflects the real problem to be solved, that the relationships among the elements have the correct direction and no important aspects of the problem have been ommited.

This process, for the generic model herein analysed, was effectuated by a group of project management experts, as to ensure its completeness and consistency. The goal was to design a generic model that could fit in a variety of project scheduling cases and would give a way to express those qualitative factors that affect the way that the project should be scheduled without omitting the quantitative data needed for the prioritisation of the objectives.

After the generation of the network describing the problem, the decision makers are asked to enter their judgments and the well-known ANP calculation steps follow to compute the priorities which in this case are the optimization objectives.

\section{Conclusions}

The project schedule generation problem seeks a baseline schedule for an upcoming project where time, cost, smooth resource profile, minimal maximum usage per resource type and robustness are the core objectives. Project managers always reason in terms of a mix of the above objectives, therefore, a multidimensional approach is implicitly or explicitly taken in practice (Viana et al, 2000). These different aspects, are often conflicting and all of them need to be taken into consideration and will play different role in the schedule generation process based on the specific organisation and its priorities, the size and the budget of the project, the customer and other environmental parameters.

The proposed multi-objective approach requires from the decision maker to select the desired optimisation objectives and the criteria and relationships among them, to be able to prioritise the objectives. The ANP is used to generate the weight vector that reflects the preferences of the decision makers. This weight vector, gives an a priori knowledge of the decision makers preferences about the optimisation objectives and is used to limit the solution search space when looking for weighted pareto optimal solutions. 


\section{REFERENCES}

Abbasi, B., Shadrokh, S., Arkat, J., 2006. Bi-objective resource-constrained project scheduling with robustness and makespan criteria. Applied Mathematics and Computation, 180, 146-152.

Alcaraz, J., Maroto, C., Ruiz, R., 2003. Solving the Multi-Mode Resource-Constrained Project Scheduling Problem with genetic algorithms. Journal of the Operational Research Society, 54, 614-626.

Ballestín, F., Valls, V., Quintanilla, S., 2008. Pre-emption in resource-constrained project scheduling. European Journal of Operational Research, 189, 1136-1152.

Bartusch, M., Möhring, R.H., Radermacher, F.J., 1988. Scheduling project networks with resource constraints and time windows. Annals of Operations Research, 16, 199-240.

Bianco, L., Caramia, M., DellÓlmo, P., Solving a preemptive project scheduling problem with coloring techniques. Wȩglarz, 193, 135-146.

Boctor, F.F., 1996. Resource-constrained project scheduling by simulated annealing. International Journal of Production Research, 34, 2335-2351.

Brucker, P., Drexl, A., Möhring, R., Neumann, K., Pesch, E., 1999. Resource-constrained project scheduling: Notation, classification, models, and methods. European Journal of Operational Research, 112, 3-41.

Buddhakulsomsiri, J., Kim, D.S., 2006. Properties of multi-mode resource-constrained project scheduling problems with resource vacations and activity splitting. European Journal of Operational Research, 175, 279-295.

Buddhakulsomsiri, J., Kim, D.S., 2007. Priority rule-based heuristic for multi-mode resource-constrained project scheduling problems with resource vacations and activity splitting. European Journal of Operational Research, 178, 374-390.

Calhoun, K.M., Deckro, R.F., Moore, J.T., Chrissis, J.W., Van Hove, J.C., 2002. Planning and re-planning in project and production scheduling. Omega, 30, 155-170.

Cesta, A., Oddi, A., Smith, S.F., 2002. A constraint-based method for project scheduling with time windows. Journal of Heuristics, 8, 109-136.

Coelho, J., Vanhoucke, M., 2011. Multi-mode resource-constrained project scheduling using RCPSP and SAT solvers. European Journal of Operational Research, 213, 73-82.

Damay, J., Quilliot, A., Sanlaville, E., 2007. Linear programming based algorithms for preemptive and nonpreemptive RCPSP. European Journal of Operational Research, 182, 1012-1022.

Davis, E.W., 1973. PROJECT SCHEDULING UNDER RESOURCE CONSTRAINTS - HISTORICAL REVIEW AND CATEGORIZATION OF PROCEDURES. AIIE Trans, 5, 297-313.

De Reyck, B., Herroelen, W., 1999. The multi-mode resource-constrained project scheduling problem with generalized precedence relations. European Journal of Operational Research, 119, 538-556.

Deblaere, F., Demeulemeester, E., Herroelen, W., 2010. Reactive scheduling in the multi-mode RCPSP. Computers and Operations Research, 38, 63-74.

Demeulemeester, E.L., Herroelen, W.S., 1996. An efficient optimal solution procedure for the preemptive resourceconstrained project scheduling problem. European Journal of Operational Research, 90, 334-348.

Hartmann, S., 2013. Project scheduling with resource capacities and requests varying with time: A case study. Flexible Services and Manufacturing Journal, 25, 74-93.

Hartmann, S., Briskorn, D., 2010. A survey of variants and extensions of the resource-constrained project scheduling problem. European Journal of Operational Research, 207, 1-14.

Heilmann, R., 2001. Resource-constrained project scheduling: A heuristic for the multi-mode case. OR Spektrum, 23, 335-357.

Heilmann, R., 2003. A branch-and-bound procedure for the multi-mode resource-constrained project scheduling problem with minimum and maximum time lags. European Journal of Operational Research, 144, 348-365.

Jarboui, B., Damak, N., Siarry, P., Rebai, A., 2008. A combinatorial particle swarm optimization for solving multimode resource-constrained project scheduling problems. Applied Mathematics and Computation, 195, $299-308$.

Józefowska, J., Mika, M., Rózycki, R., Waligóra, G., Wȩglarz, J., 2001. Simulated Annealing for Multi-Mode Resource-Constrained Project Scheduling. Annals of Operations Research, 102, 137-155.

Kimms, A., 2001. Maximizing the Net Present Value of a Project under Resource Constraints Using a Lagrangian Relaxation Based Heuristic with Tight Upper Bounds. Annals of Operations Research, 102, 221-236.

Kobylański, P., Kuchta, D., 2007. A note on the paper by M. A. Al-Fawzan and M. Haouari about a bi-objective problem for robust resource-constrained project scheduling. International Journal of Production Economics, 107, 496-501. 
Kolisch, R., 2000. Integrated scheduling, assembly area- and part-assignment for large-scale, make-to-order assemblies. International Journal of Production Economics, 64, 127-141.

Kolisch, R., Drexl, A., 1997. Local for multi-mode resource-constrained project. IIE Transactions (Institute of Industrial Engineers), 29, 987-999.

Kyriakidis, T.S., Kopanos, G.M., Georgiadis, M.C., 2012. MILP formulations for single- and multi-mode resourceconstrained project scheduling problems. Computers \&amp; Chemical Engineering, 36, 369-385.

Lorenzoni, L.L., Ahonen, H., Alvarenga, A.G.d., 2006. A multi-mode resource-constrained scheduling problem in the context of port operations. Computers and Industrial Engineering, 50, 55-65.

Mika, M., Waligóra, G., Weȩglarz, J., 2005. Simulated annealing and tabu search for multi-mode resourceconstrained project scheduling with positive discounted cash flows and different payment models. European Journal of Operational Research, 164, 639-668.

Mika, M., Waligóra, G., Weglarz, J., 2008. Tabu search for multi-mode resource-constrained project scheduling with schedule-dependent setup times. European Journal of Operational Research, 187, 1238-1250.

Neumann, K., Schwindt, C., Zimmermann, J., Resource-constrained project scheduling with time windows: Recent developments and new applications. Józefowska, Wȩglarz, 95, 375-407.

Neumann, K., Schwindt, C., Zimmermann, J., 2002. Recent results on resource-constrained project scheduling with time windows: Model, solution methods, and applications. Central European Journal of Operations Research, 10, 113-148.

Neumann, K., Schwindt, C., Zimmermann, J., 2003. Project Scheduling with Time Windows and Scarce Resources: Temporal and Resource-Constrained Project Scheduling with Regular and Nonregular Objective Functions. Springer.

Neumann, K., Zimmermann, J., Methods for resource-constrained project scheduling problem with regular and nonregular objective functions and schedule-dependent time windows. Wȩglarz, 193, 261-288.

Neumann, K., Zimmermann, J., 2000. Procedures for resource leveling and net present value problems in project scheduling with general temporal and resource constraints. European Journal of Operational Research, 127, 425443.

Peteghem, V.V., Vanhoucke, M., 2010. A genetic algorithm for the preemptive and non-preemptive multi-mode resource-constrained project scheduling problem. European Journal of Operational Research, 201, 409-418.

Saaty, T.L., 1996. Decision making with dependence and feedback: the analytic network process. RWS Publications, Pittsburgh.

Sabzehparvar, M., Seyed-Hosseini, S.M., 2008. A mathematical model for the multi-mode resource-constrained project scheduling problem with mode dependent time lags. Journal of Supercomputing, 44, 257-273.

Seifi, M., Tavakkoli-Moghaddam, R., 2008. A new bi-objective model for a multi-mode resource-constrained project scheduling problem with discounted cash flows and four payment models. International Journal of Engineering, Transactions A: Basics, 21, 347-360.

Talbot, F.B., 1982. RESOURCE-CONSTRAINED PROJECT SCHEDULING WITH TIME-RESOURCE TRADEOFFS: THE NONPREEMPTIVE CASE. MANAGE SCI, V 28, 1197-1210.

Ulusoy, G., Sivrikaya-Şerifoğlu, F., Şahin, Ş., 2001. Four Payment Models for the Multi-Mode Resource Constrained Project Scheduling Problem with Discounted Cash Flows. Annals of Operations Research, 102, $237-$ 261.

Vanhoucke, M., Coelho, J., Debels, D., Maenhout, B., Tavares, L.V., 2008. An evaluation of the adequacy of project network generators with systematically sampled networks. European Journal of Operational Research, 187, $511-524$. Vanhoucke, M., Demeulemeester, E., Herroelen, W., 2001. An Exact Procedure for the Resource-Constrained Weighted Earliness-Tardiness Project Scheduling Problem. Annals of Operations Research, 102, 179-196.

Viana, A., Pinho de Sousa, J., 2000. Using metaheuristics in multiobjective resource constrained project scheduling. European Journal of Operational Research, 120, 359-374.

Voß, S., Witt, A., 2007. Hybrid flow shop scheduling as a multi-mode multi-project scheduling problem with batching requirements: A real-world application. International Journal of Production Economics, 105, 445-458. 